\title{
Cascade: An Epidemiological Model to Simulate Disease Spread and Aggregation Across Multiple Scales in a Spatial Hierarchy
}

\author{
M. Gosme and P. Lucas
}

UMR1099, Biologie des Organismes et des Populations appliquée à la Protection des Plantes, INRA-Agrocampus, F-35653 Le Rheu. Current address of first author: UMR211 Agronomie, INRA-AgroParisTech, 78850 Thiverval-Grignon, France. Accepted for publication 17 October 2008.

\section{ABSTRACT}

Gosme, M., and Lucas, P. 2009. Cascade: An epidemiological model to simulate disease spread and aggregation across multiple scales in a spatial hierarchy. Phytopathology 99:823-832.

Disease spread occurs at several spatial scales, e.g., from field to field, plant to plant, and leaf to leaf. So far, epidemiological models have largely overlooked the multiscale nature of epidemics. Here, we propose a model that simulates disease spread across multiple scales in a nested hierarchy. The model is based on the central ideas of hierarchy theory, i.e., (i) the system is decomposed vertically into levels and horizontally into holons (elements at one level, which are complete systems when seen from the lower level), and (ii) higher levels are characterized by slower processes than lower levels. The model is individual-based, the individuals being the holons, which are either susceptible or infected. At each level, infections within one holon (i.e., infections between holons of the level below) occur independently from the other holons: infections be- tween holons happen at the higher level. The self-similarity of the model structure and processes across all levels allows implementing the model with a simple recursive algorithm. The behavior of the model was studied using methods commonly applied to field data. Aggregation of the disease was characterized through the incidence-incidence relationship and the binomial power law, in order to study the effect of infectiousness at each level on disease aggregation. Sensitivity analyses showed that disease incidences at all levels were influenced by the infectiousness at any level, but infectiousness at higher levels had more effect than infectiousness at lower levels. It was also shown that increasing the probability of infection at a given level increased aggregation at higher level(s) and decreased aggregation at lower level(s). The results were consistent between incidence-incidence relationship and power law analysis, but the incidence-incidence relationship was more sensitive in detecting the differences in aggregation between treatments.
One of the primary scopes of plant disease epidemiology, both practical and theoretical, has long been the temporal development of epidemics. To this aim, classical growth curves (e.g., monomolecular or logistic growth) have proven very useful, despite their inherent shortcomings. Indeed, they suppose that the susceptible and infected individuals (or inoculum) mix perfectly and that the per capita infection rate and total host population are constant. These assumptions are known to be wrong (5) but the models nonetheless remain useful as they allow us to think about epidemics (complex systems involving numerous processes) in terms of three components when comparing epidemics or assessing the effectiveness of control methods: the mixing of infective and susceptible individuals, the relative rate of infection, and the carrying capacity. However, there are cases where a more detailed description of reality is needed and much work has been devoted to the incorporation of more biological realism into epidemiological models, in particular disease heterogeneity and spatial patterns (27).

The spatial dimension can be explicitly introduced into epidemiological models, usually by considering the pathogen's spread over a lattice of cells or patches $(8,17,40)$. The spatial component of epidemics can also be introduced implicitly by incorporating spatial information in temporal development models, either by correcting the logistic curve $(21,26,34,42)$ or by more complex statistical models tracking both the dynamics of mean disease

Corresponding author: M. Gosme: E-mail address: marie.gosme@grignon.inra.fr

* The $\boldsymbol{e}$-Xtra logo stands for "electronic extra" and indicates that the online version contains an algorithm showing how the program walks through the "tree" of hierarchical levels at each time step.

doi:10.1094/PHYTO-99-7-0823

(C) 2009 The American Phytopathological Society level and spatial correlation between individuals and making necessary approximations such as pair approximation (7), moment closure (3), or Kirkwood approximation (9).

However, these models only consider one spatial entity: individuals which are either susceptible or infected (there may be other categories such as latent or removed but the individual is always considered as a whole). In many cases, this could be seen as an over-simplification: individuals (e.g., plants) are actually made of smaller parts (e.g., leaves), which can be healthy or diseased and are, in turn, made of even smaller parts (e.g., leaflets). Indeed, complex systems such as biological systems are generally organized in hierarchically nested levels: leaves grow on twigs; twigs are grouped into branches; branches form trees; trees make forests, and so on.

The hierarchical structure of epidemics has been underlined early in the history of botanical epidemiology, e.g., country> field $>$ foci $>$ plant $>$ lesion (33). Futhermore, epidemiological data at several hierarchical levels are available for some pathosystems. For example, the incidence of strawberry leaf blight at four levels: field $>$ row $>$ leaf $>$ leaflet (30), or the incidence of Fusarium head blight at two levels: ear>spikelet (41). A strong relationship between canopy structure and disease aggregation has been detected in strawberry leaf blight (31) or rice sheath blight $(25,38)$. Recently, there has been a growing interest in the relationship between incidences at different hierarchical levels $(16,22,32)$. Indeed, this relationship captures important aspects for the description of epidemics, although the underlying processes responsible for these patterns are often not well understood (22).

From such a description, a further step would then be to understand the processes responsible for the observed relationships and to model them. Until recently, there has been a scarcity of approaches that incorporate both spatial and temporal aspects as 
well as patch structure for plant disease (31). Epidemiologists are beginning to fill this gap and a few models that take into account the hierarchical nature of epidemiological systems have been developed, in botanical epidemiology (36) or in human epidemiology (35), but none uses hierarchy theory in order to model the system's dynamics.

Hierarchy theory provides a theoretical framework that allows handling complex systems. Wu and David (39) defined a hierarchical approach as a way of breaking down complexity and a process of discovering or rendering order. The first step of this approach is to derive the hierarchical organization from differences in temporal and spatial scales between the phenomena of interest (23). Correctly defined, hierarchical levels allow the delimiting of holons (i.e., the elements of the system). A holon is both an atomic whole, when seen from a given level of the hierarchy, and a compound, when seen at a lower level of the hierarchy (i.e., higher spatial and/or temporal resolution) (18). At each level, holons are defined in such a manner that the interactions between their components are significantly more frequent within than between holons. For example, an epidemic developing in a landscape can be described by the spread of the disease between fields at the higher level and the spread of the disease from plant to plant at the level below, with stronger interactions between plants from the same field than between plants from different fields, and so on.

The objective of this paper is to present a modeling approach that allows the use of spatially implicit epidemiological models in a hierarchical perspective, in order to simulate disease dynamics across a range of hierarchical levels.

\section{THEORY AND APPROACHES}

General structure. The model presented here was built in order to simulate different levels in a nested spatial hierarchy, each level being characterized by its dispersal process. The model is individual-based, the individuals being the holons of each level in a spatial hierarchy (e.g., fields, plants, roots or leaves, and sites). Each individual is either susceptible or infected. In the following, when the explanation involves several levels (e.g., plant $>$ leaf $>$ site), the individuals at a given level (level $\lambda$ ) will be referred to as a holon (e.g., leaf), its components (level $\lambda-1$ ) will be termed "elements" (e.g., sites) and the holon at the upper level (level $\lambda+1$ ) will be called "group" (e.g., plant), although of course all holons can be groups and elements, depending on the level of interest. The term "incidence" refers to the proportion of infected holons at a given level, across all groups, and "conditional incidence" refers, in one group, to the proportion of infected elements conditional to the holons being infected (i.e., number of infected elements divided by total number of elements in infected holons).

By the definition of a holon, interactions between holons are significantly more frequent within than between groups. More specifically, the model relies on the assumption that the host's spatial structure is sufficiently strong (or the infection processes at different levels sufficiently different) so that interactions between holons from two different groups are negligible compared with interactions between holons of the same group, i.e., once a group is infected on one of its holons, infection of its other holons only arises from secondary infection among these holons. This assumption greatly simplifies the model. At each level, infection of holons in one group occurs independently from the other groups and in turn, infection of elements in one holon occurs independently from the other holons. Note that this is essentially the same assumption of a closed population which underlies growth curves models.

Each level is influenced by the level immediately above, through primary infections (i.e., the infection of the first holon when a group becomes infected), and from the level immediately below, through a link function which relates the probability of infection with the conditional incidence at the element level, so that "infectiousness" of diseased holons will increase if they are severely infected (Fig. 1; Table 1 for a list of all variables used in the model).

Infection process. Mixing term. Infection occurs in two ways: the first holon (or first $\delta_{\lambda}$ holons) of a previously susceptible group becomes infected when the group becomes infected.

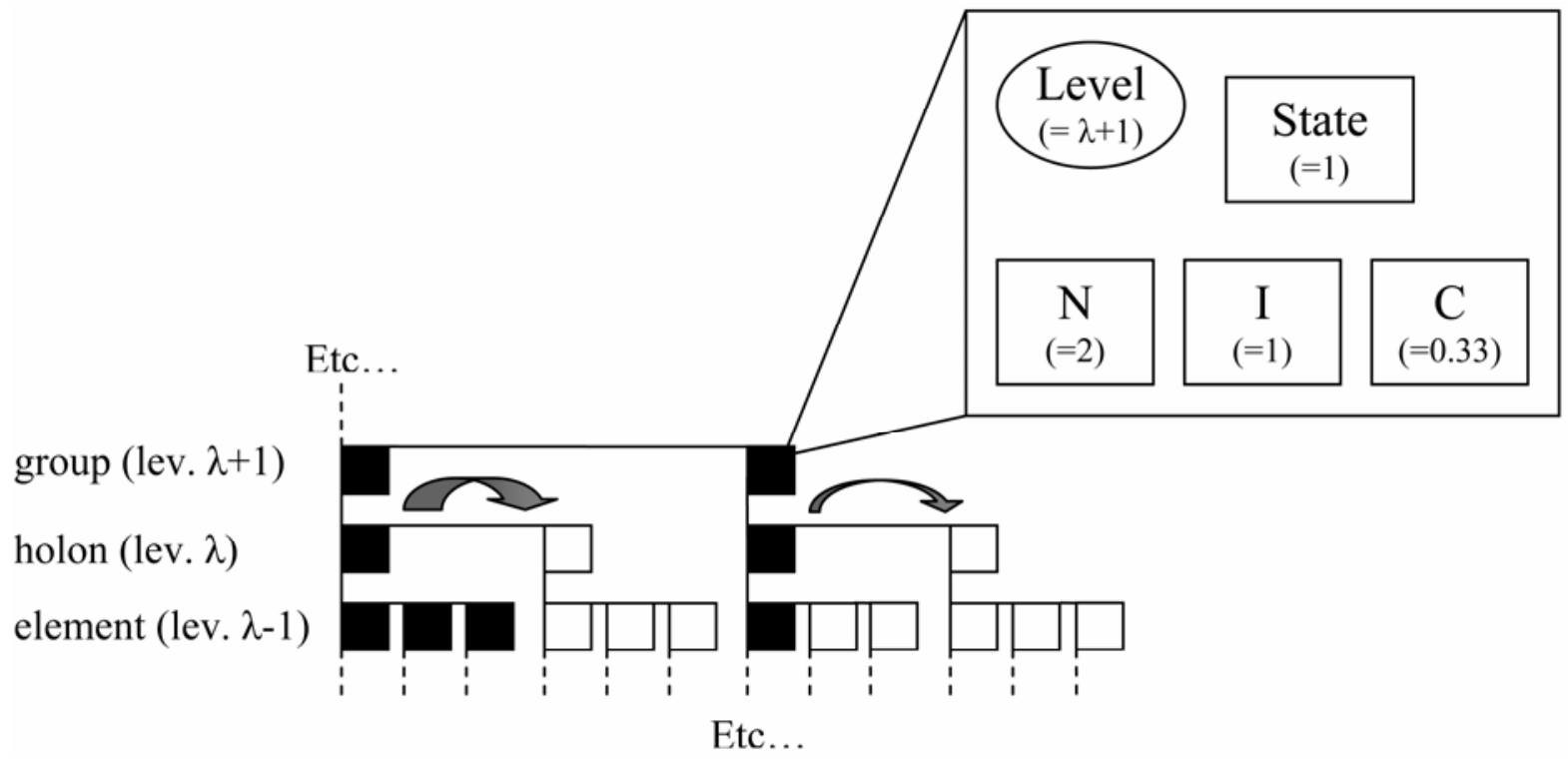

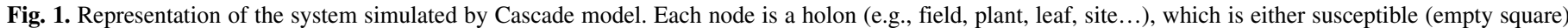

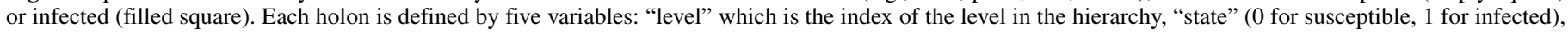

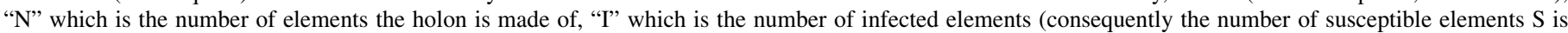

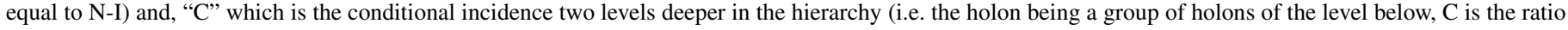

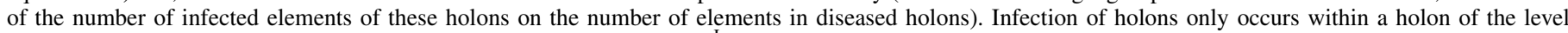

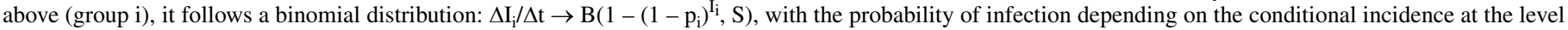

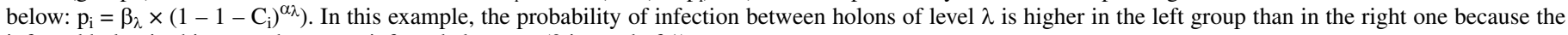
infected holon in this group has more infected elements ( 3 instead of 1). 
Subsequent holons become infected through secondary infection between the infected and susceptible holons of this group. The mixing term for secondary infection can be different for each level, in order to reflect the spatial distribution of the holons at this level. For example, perfect mixing can be used in order to represent a nondimensional system; a constant probability of infection can be used to represent a one-dimensional system with contact spread; the probability of infection can be made proportional to the square root of the number of infected holons in order to represent two-dimensional spread of circular foci; or any other suitable mixing term can be specified.

Only perfect mixing will be used in this paper. At each time step and in each group $i$ of level $\lambda+1$, with $S_{i}$ susceptible holons and $I_{i}$ infected ones, the number of new infections follows a binomial distribution (B):

$$
\frac{\Delta \mathrm{I}_{\mathrm{i}}}{\Delta \mathrm{t}} \rightarrow \mathrm{B}\left(1-\left(1-\mathrm{p}_{\mathrm{i}}\right)^{\mathrm{I}_{\mathrm{i}}}, \mathrm{S}_{\mathrm{i}}\right)
$$

where $\mathrm{p}_{\mathrm{i}}$ is the probability of infection between a susceptible and an infected holon in group $\mathrm{i}$ :

$$
\mathrm{p}_{\mathrm{i}}=\mathrm{f}_{\lambda}\left(\beta_{\lambda}, \mathrm{C}_{\mathrm{i}}\right)
$$

where $\beta_{\lambda}$ is the infectiousness of a totally infected holon (level $\lambda$ ); $\mathrm{C}_{\mathrm{i}}$ is the conditional incidence at the element level, i.e., ratio of the number of infected elements in group $i$ on the number of elements in diseased holons of group $\mathrm{i} ; \mathrm{f}_{\lambda}$ is a link function between conditional incidence at the element level $(\lambda-1)$ and probability of infection between holons (level $\lambda$ ), such that $p_{i}$ increases from 0 to $\beta_{\lambda}$ when $C_{i}$ increases, i.e., the more severely infected the holons are, the more likely they are to transmit the infection to other holons.

Link function. To allow for different behaviors of the relationship between conditional incidence at the element level and probability of infection at the holon level, the following link function is used:

$$
\mathrm{f}_{\lambda}\left(\beta_{\lambda}, \mathrm{C}_{\mathrm{i}}\right)=\beta_{\lambda} \times\left(1-\left(1-\mathrm{C}_{\mathrm{i}}\right)^{\alpha_{\lambda}}\right)
$$

where $\beta_{\lambda}$ is the infectiousness of a totally infected holon (level $\lambda$ ), $\mathrm{C}_{\mathrm{i}}$ is the mean incidence at the element level (level $\lambda-1$ ) among diseased holons (level $\lambda$ ) and $\alpha_{\lambda}$ is a parameter determining the shape of the relationship specific to level $\lambda$. Thus the model can represent a large range of pathosystems, from almost systemically transmitted diseases (large $\alpha$ ) to diseases which are transmitted only by heavily infected holons (small $\alpha$ ). By default, $\alpha_{\lambda}=1$ and the infectiousness is simply corrected by the conditional incidence.

Host growth. At each level, it is possible to choose between no host growth, monomolecular, and logistic growth functions. At each time step and for each group $\mathrm{i}$ of level $\lambda+1$ with $\mathrm{N}_{\mathrm{i}}$ holons, the rate of creation of new holons follows a Poisson distribution (P):

monomolecular growth:

$$
\frac{\Delta \mathrm{N}_{\mathrm{i}}}{\Delta \mathrm{t}} \rightarrow \mathrm{P}\left(\gamma_{\lambda} \times\left(1-\frac{\mathrm{N}_{\mathrm{i}}}{\mathrm{v}_{\lambda}}\right)\right)
$$

logistic growth:

$$
\frac{\Delta \mathrm{N}_{\mathrm{i}}}{\Delta \mathrm{t}} \rightarrow \mathrm{P}\left(\gamma_{\lambda} \times \mathrm{N}_{\mathrm{i}} \times\left(1-\frac{\mathrm{N}_{\mathrm{i}}}{v_{\lambda}}\right)\right)
$$

where $\gamma_{\lambda}$ is the intrinsic rate of growth and $v_{\lambda}$ is the maximum number of holons (level $\lambda$ ) per group (level $\lambda+1)$.

Model implementation. The self-similarity of the model structure and processes across hierarchical scales allows using a simple recursive algorithm to implement the model: the system is represented as a tree, each node being one holon, with vertical branches (from the holon to the first of its elements) and horizontal branches (from the holon to the next, within a group). At each time step, the tree is walked from the top level of the hierarchy to the bottom level, going first down vertical branches and then right along horizontal branches. This allows transfer of the primary infections (i.e., the infection of the first holon when a group becomes infected) immediately from the upper levels to the lower levels. Conversely, host growth and secondary infections are computed based on information collected at the end of the previous time step. A simplified presentation of this algorithm is given in the annex. The model is programmed in $\mathrm{C}++$, the executable file as well as source files can be obtained from the authors.

The output variables for each level are as follows: $\mathrm{N}_{\lambda}$ is the total number of holons (sum across all groups); $\mathrm{I}_{\lambda}$ is the number of diseased holons (sum across all groups); and $\mathrm{V}_{\mathrm{obs} \lambda}$ is the variance (across all groups) of the number of diseased holons per group.

Description of the simulations. Model verification. In order to verify the behavior of the model, an arbitrary 3-level system was simulated, with 1,000 holons at the highest level (level 3, e.g., plant), 25 holons (in each group) at the intermediate level

TABLE 1. Definition and unit of the variables used in the model Cascade

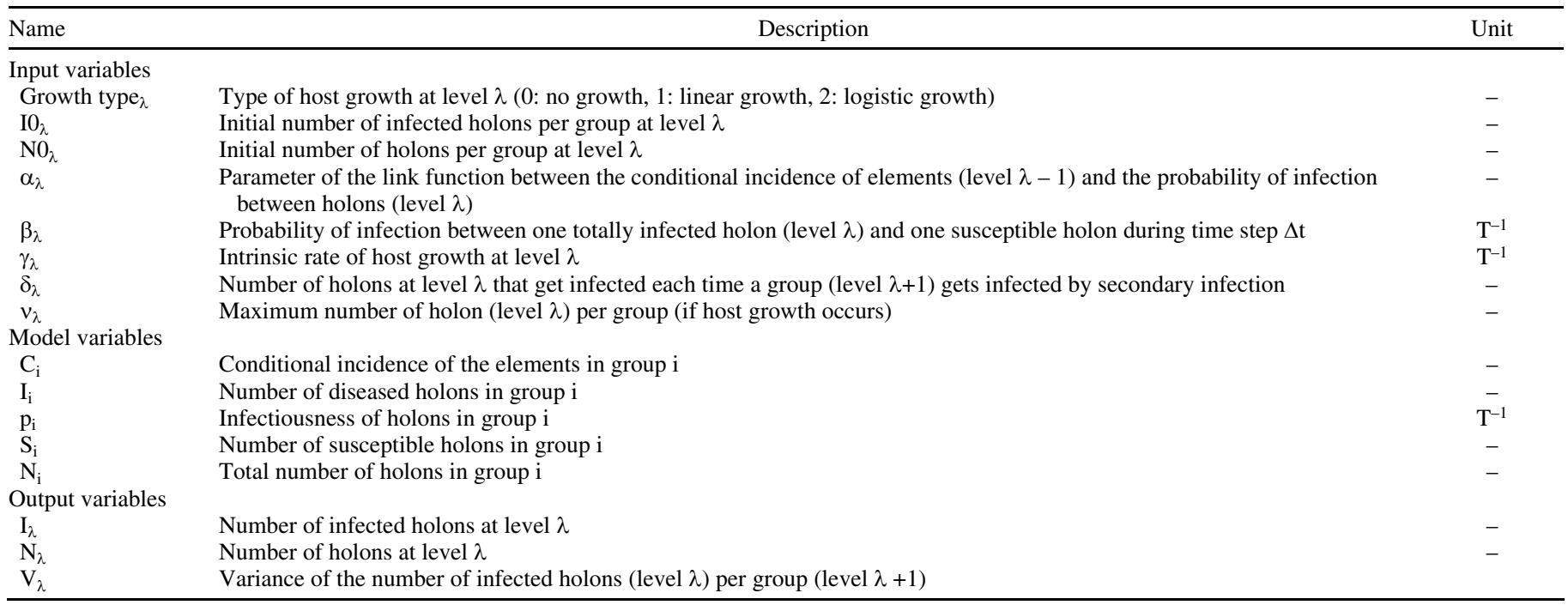


(level 2, e.g., leaf) and 49 holons (in each group) at the lowest level (level 1, e.g., site). The number of holons at each level was kept constant: no host growth occurred. Epidemics were initiated with a single infected holon at the lowest level. The maximum infectiousness $\left(\beta_{\lambda}\right)$ was $2 \mathrm{E}-4,2 \mathrm{E}-2$, and $2 \mathrm{E}-2$ at the level 3,2 , and 1 , respectively. These values were chosen so that the transition time at the intermediate level, i.e., the time needed to go from having one infected element (holon at the lowest level) to all but one infected elements, would be approximately 10 time steps (e.g., days) and the complete epidemics would develop in approximately 150 days. The parameter of the link function was equal to one at each level. The simulations were run for 300 time steps (e.g., days) and 100 repetitions were done in order to obtain a distribution for the results.

Sensitivity analysis. The simulations for the sensitivity analysis were performed on the same 3-level system as the one used for model verification and the same parameter values were used as a reference. The effect of the simultaneous variation of the infectiousness of totally infected holons $(\beta)$ at all three levels was first analyzed with a complete factorial design with two values (50 and $100 \%$ of the reference value) for each of the three parameters $\left(\beta_{1}, \beta_{2}\right.$, and $\left.\beta_{3}\right)$. The effect of each parameter was then individually analyzed by varying the parameter between 50 and $125 \%$ of its reference value while the other two parameters were set to their reference value. The simulations were run for 300 time steps and 100 repetitions were done for each set of parameters.

The effect of the parameter of the link function $\left(\alpha_{\lambda}\right)$ was studied with a complete factorial design with three values $(0.1,1$, and 10) for each of the two parameters $\left(\alpha_{3}\right.$ and $\left.\alpha_{2}\right)$. The simulations were run for 300 time steps and 100 repetitions were done for each set of parameters.

Data analysis. The areas under disease progress curves (AUDPC) at each level were computed between time steps 0 and 299. The normality of the distribution of AUDPC was tested with a Shapiro-Wilk test (the significance level was modified according to the Bonferroni correction to control experiment-wise error rate below 5\%) using the $\mathrm{R}$ statistical software. The distributions of AUDPC obtained at each level with different parameter values

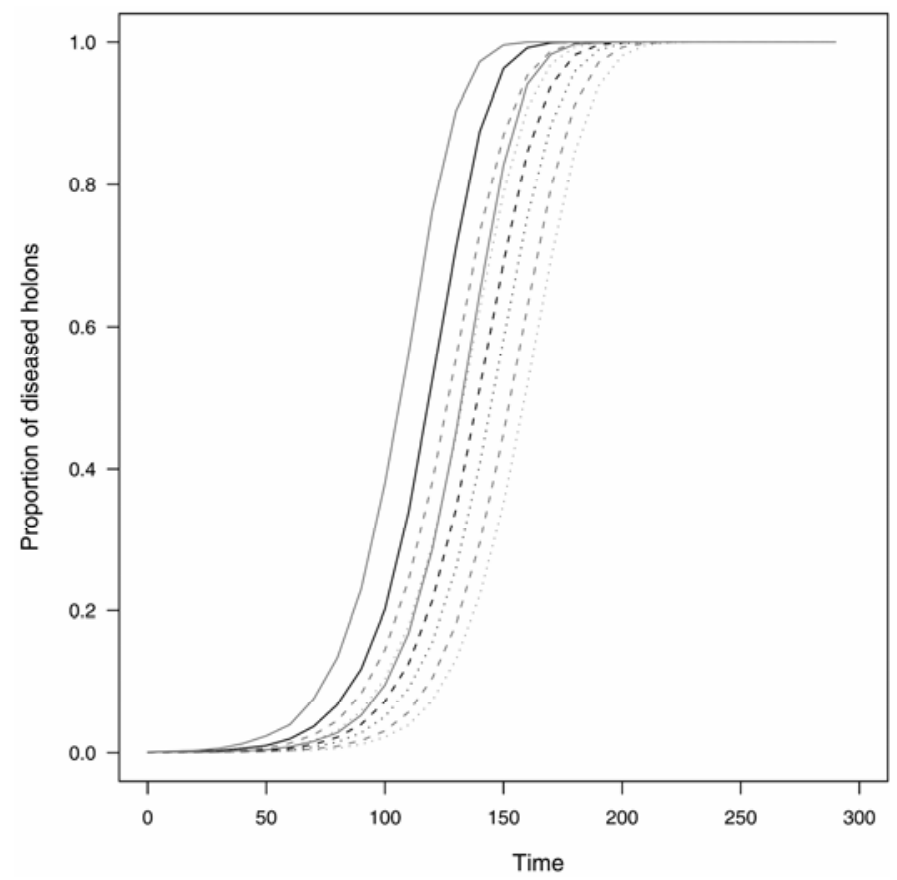

Fig. 2. Evolution of the proportion of diseased holons at three scales in a spatial hierarchy as simulated by Cascade model. Solid line: level 3 (highest level), dashed line: level 2 (intermediate level), and dotted line: level 1 (lowest level). Black line: median value, gray lines: 5 and $95 \%$ quantiles. were compared with a Kolmogorov-Smirnov test, first between the reference value and 50,75 , and $125 \%$ of the reference value for parameters $\beta_{1}, \beta_{2}$, and $\beta_{3}$ (number of tests considered for the Bonferroni correction $=27$ ) and second between the reference value and the eight variations of $\alpha_{2}$ and $\alpha_{3}$ parameters (number of tests considered for the Bonferroni correction $=24$ ).

The relationship between incidences at two successive levels in the hierarchy $\left(\mathrm{I}_{\text {high }}\right.$ and $\mathrm{I}_{\text {low }}$ ) was fitted with the following equation $(15,19,22)$ :

$$
\mathrm{I}_{\text {high }}=1-\left(1-\mathrm{I}_{\text {low }}\right)^{\mathrm{c}}
$$

As c approaches 1, diseased holons are more aggregated within infected groups. The c parameter was estimated for each simulation with nonlinear least squares using $\mathrm{R}$ statistical software. Normality of the distribution of $\mathrm{c}$ and comparison between parameter values were carried out in the same way as in the case of the AUDPC.

The binary form of Taylor's (29) power law $(14,20)$ was used to describe the relationships between the observed and theoretical (binomial) variance of disease incidence at each level.

$$
\ln \left(\mathrm{V}_{\text {obs }}\right)=\mathrm{a}+\mathrm{b} \times \ln \left[\mathrm{n} \times \mathrm{I}_{\text {low }} \times\left(1-\mathrm{I}_{\text {low }}\right)\right]
$$

where $\mathrm{V}_{\mathrm{obs}}$ is the observed (in our case simulated) variance of the number of diseased holons per group, $\mathrm{n}$ is the number of holons per group ( $n=25$ at level 2 and $n=49$ at level 1$)$ and a and $b$ are the intercept and slope of a straight line, respectively. A slope greater than one indicates that disease heterogeneity increases systematically with mean incidence; a slope equal to one with an intercept greater than zero indicates a constant aggregation of the disease (14). Since the power law relationship showed a succession of line segments, the linear relationship described by equation 7 was estimated for each segment separately. The linear regression was performed using the $\mathrm{R}$ statistical software. Normality of the distribution of the intercept was tested with a Shapiro-Wilk test and comparison between parameter values were carried out in the same way as in the case of the AUDPC.

\section{RESULTS}

Model verification. Disease curves. The evolution of the proportion of diseased holons in the case of a 3-level system (Fig. 2) shows that the incidences at all three levels followed sigmoid curves. For each pair of successive levels, the incidence at the higher level was higher than the incidence at the lower level; however, $I_{1}$ was very close to $I_{2}$, indicating that disease intensified rapidly within holons of level 2 , resulting in a strong aggregation of diseased elements (level 1) within holons (level 2).

Incidence-incidence relationship. The incidence-incidence relationship (Fig. 3) confirmed these results. The curve relating incidence at level 2 with incidence at level 1 was close to the first bisector (mean estimated c was 1.37 , sd $=0.07$ ) i.e., aggregation of diseased elements within holons was almost maximal. Diseased holons (level 2) were less aggregated within groups (level 3 ), but still aggregated: the mean of estimated c was 3.04 ( $\mathrm{sd}=$ 0.009 ) while it would have been 25 if the distribution of diseased holons among groups had been random, because there were $n=$ 25 holons per group.

Power law. The power law relationship is plotted in Figure 4. The logarithm of the simulated variance did not lie on a straight line when plotted against the logarithm of the theoretical (random) variance. Four different phases, each corresponding to a straight line, could be differentiated. At the beginning of the epidemic, the simulated variance increased more than the theoretical variance, resulting in a slope greater than one; for the variance of the number of infected holons at level 2, this phase lasted about 38 days, until incidence at level 2 was approximately 
0.002 , for the variance of the number of infected holons at level 1 , it lasted about 12 days, until incidence at level 1 was approximately 7.4E-5). In the second phase, the slope was equal to one, indicating a constant level of aggregation, independent of the mean disease level. When disease incidence reached 0.5 , there was a cusp, after which the logarithm of the theoretical and simulated variance started to decrease. The third phase thus corresponded to a phase where the logarithm of the simulated variance decreased at almost the same rate as the logarithm of the binomial variance, resulting in a slope only slightly greater than one (1.12 in the case of the variance of the number of diseased holons at the intermediate level and 1.03 in the case of holons at the lowest level). In the sensitivity analysis, phases two and three were pooled in order to compute the intercept when aggregation was constant. The fourth segment corresponded to a phase when the simulated variance decreased more quickly than the theoretical variance. The dots corresponding to phase four sit on the same line as phase one, because the number of holons at each level is constant during the whole simulation as well as the infection rates, so the last infected holons get depleted of susceptible elements at the same rate as the elements were getting infected in the first infected holons.

Sensitivity analysis. Maximum infectiousness. Disease incidence at all three levels was influenced by all three maximum infectiousness parameters ( $\beta$ ) (Fig. 5), but the infectiousness at the highest level had more effect on all three levels than the infectiousness at the intermediate and lowest level.

The interactions between the infectiousness at different levels were not significant (data not shown), so the effect of each parameter was analyzed separately. An increased $\beta$ at any level resulted in an increase in AUDPC at all three levels (Table 2). The Kolmogorov-Smirnov test showed that the distributions of AUDPC obtained for all tested values of all parameters at all levels differed from the distribution obtained for the reference value,

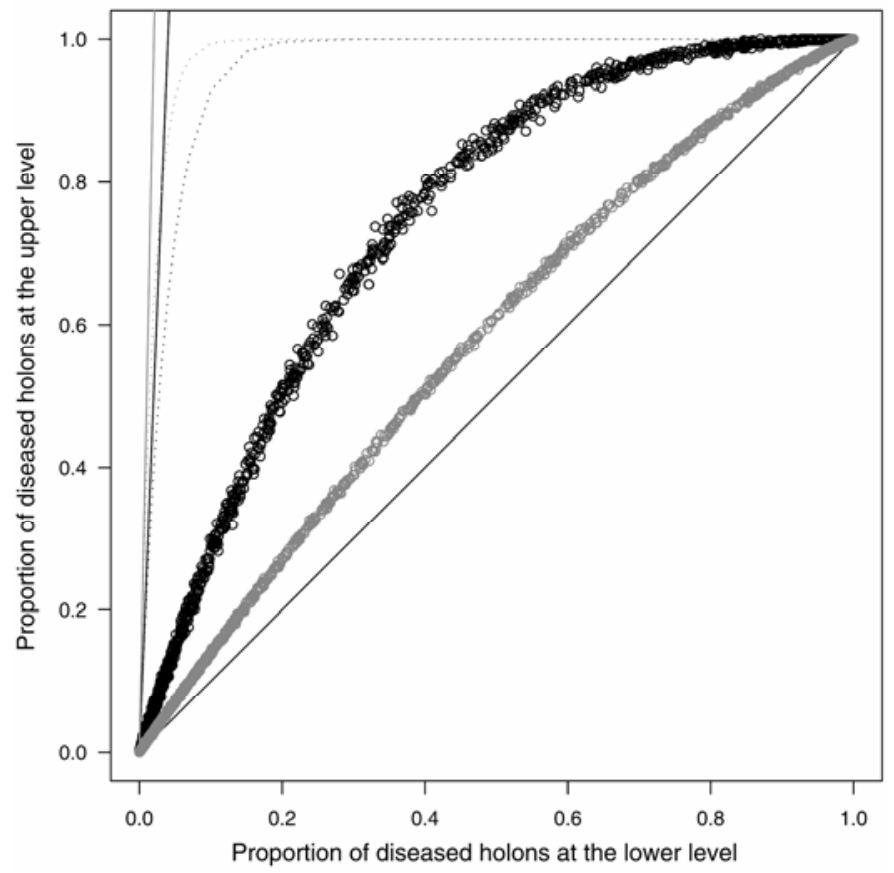

Fig. 3. Incidence-incidence relationships in epidemics simulated by Cascade model on three levels in a spatial hierarchy. Black: relationship between disease incidences at levels 3 (highest level) and 2 (intermediate level), mean c parameter (standard deviation): 3.04 (0.07), gray: relationship between disease incidences at levels 2 and 1 (lowest level) (mean c parameter: 1,37 (0.009). Dots: observed data, first bisector: line of maximum aggregation, dotted lines: theoretical relationships under the hypothesis of a random distribution $\left(\mathrm{c}_{\mathrm{th}}=\right.$ 29 and $c_{\text {th }}=49$ for levels 3-2 (black) and 2-1 (gray), respectively), solid lines: lines of maximum uniformity for level 3-2 (black) and 2-1 (gray). except in one case (difference between 100 and $125 \%$ for $\beta$ at the lowest level, when incidence was observed at the highest level).

The infectiousness also had an effect on disease aggregation, as shown by the changes in parameter $\mathrm{c}$ of the incidence-incidence relationship and the intercept of the power law relationship corresponding to the phase of constant aggregation (Fig. 6). Increasing the maximum infectiousness at the highest level $\left(\beta_{3}\right)$ decreased aggregation (higher $\mathrm{c}$ and lower intercept) of both diseased holons of the intermediate level and diseased holons of the lowest level. Increasing maximum infectiousness at the intermediate level $\left(\beta_{2}\right)$ increased aggregation (lower $\mathrm{c}$ and higher intercept) of diseased holons of the intermediate level but decreased aggregation of diseased holons of the lowest level. Increasing maximum infectiousness at the lowest level $\left(\beta_{1}\right)$ increased aggregation of both diseased holons of the intermediate level and diseased holons of the lowest level. There was a good agreement between the results of the incidence-incidence and power law analyses, but the distribution of the $\mathrm{c}$ parameter was narrower than the distribution of the intercept of the power law line (relative to the variability between treatments). Indeed, the distributions overlap on the y axis (intercept of the power law) but less so on the $\mathrm{x}$ axis (estimated $\mathrm{c}$ of the incidence-incidence relationship) in Figure 6. This resulted in the distributions of estimated $\mathrm{c}$ for both pairs of levels being different between the reference value and 50,75 , and $125 \%$ for all $\beta$, while the distribution of the intercept of the power law at level 1 was not significantly different between 75 and $100 \%$ of $\beta_{2}$, and the distribution of the intercept of the power law at level 2 was not significantly different between 125 and $100 \%$ of $\beta_{1}$.

Parameters of the link function. The parameter of the link function $\left(\alpha_{\lambda}\right)$ had a strong effect on disease incidence at all three levels (Fig. 7). The Kolmogorov-Smirnov test showed that the distributions of AUDPC obtained with different $\alpha_{1}$ and $\alpha_{2}$ were always different from the distribution of AUDPC when $\alpha_{2}$ and $\alpha_{3}$ were both equal to one. The parameter of the link function between conditional incidence at the intermediate level and infec-

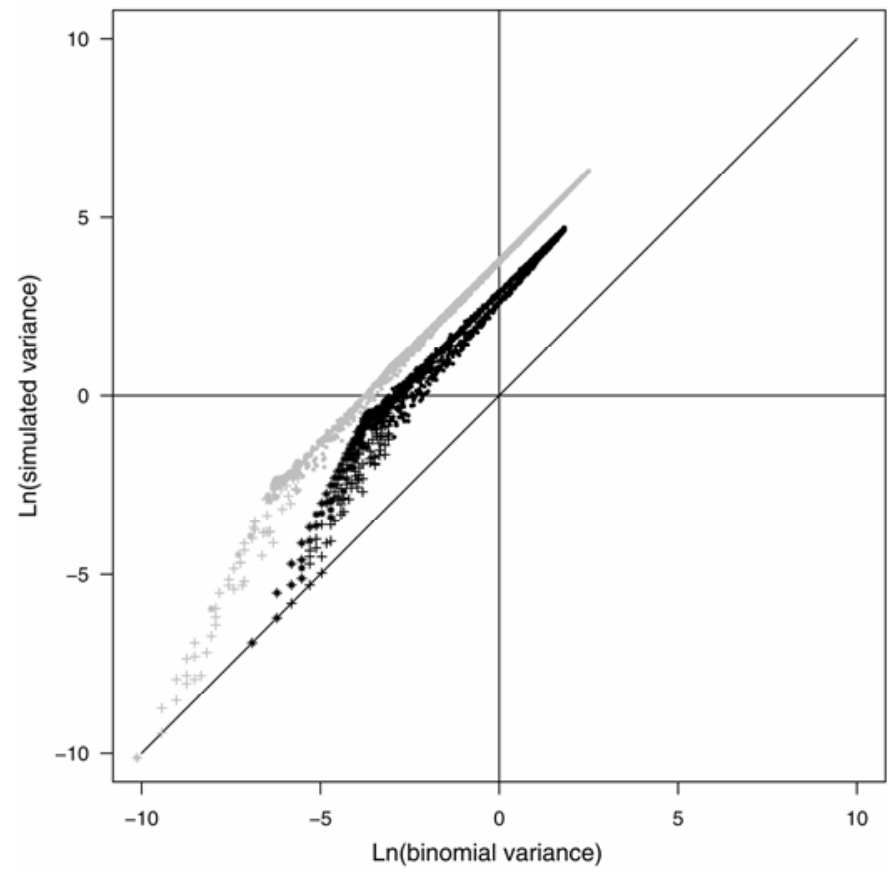

Fig. 4. Relationship between the simulated variance of the number of diseased holons per group and the binomial variance (i.e., under the hypothesis of a random distribution of diseased holons among all groups) in epidemics simulated by Cascade model on three levels in a spatial hierarchy. Black: variance of the number of diseased holons of level 2 (intermediate level); gray: variance of the number of diseased holons of level 1 (lowest level). Stars: phase 1; dots: phases 2 and 3; crosses: phase 4 (described in text). 
tion at the highest level $\left(\alpha_{3}\right)$ had more effect on the AUDPC than the parameter of the link function between conditional incidence at the lowest level and infection at the intermediate level $\left(\alpha_{2}\right)$ (Table 3). For the incidence at the highest level, the ranks were determined by the value of $\alpha_{3}$ and then the value of $\alpha_{2}$, whereas for the incidence at the intermediate and lowest levels, the combination $\left(\alpha_{3}=10\right.$ and $\left.\alpha_{2}=0.1\right)$ resulted in a smaller AUDPC than the combination $\left(\alpha_{3}=1\right.$ and $\left.\alpha_{2}=10\right)$.

The parameters of the link function also had an effect on disease aggregation, as shown by the changes in estimated $\mathrm{c}$ of the incidence-incidence relationship (Table 4) and the intercept of the power law relationship corresponding to the phase of constant aggregation (Table 5). The distributions of both $\mathrm{c}$ and intercept obtained with different $\alpha_{1}$ and $\alpha_{2}$ were always different from the distributions when $\alpha_{2}$ and $\alpha_{3}$ were equal to one. Increasing the parameter of the link function between conditional incidence at the intermediate level and infection at the highest level $\left(\alpha_{3}\right)$ decreased aggregation (higher $\mathrm{c}$ and lower intercept) of both diseased holons of the intermediate level and diseased holons of the lowest level, even more so for small values of $\alpha_{2}$. Increasing $\alpha_{2}$ increased aggregation (lower $c$ and higher intercept) of diseased holons of the intermediate level only when $\alpha_{1}$ was high. Increasing $\alpha_{2}$ decreased aggregation of diseased holons of the lowest level.

\section{DISCUSSION}

The model presented here allows for the simulation of epidemics showing different levels of aggregation at several levels in a spatial hierarchy. It can be seen as an extension of the meta-
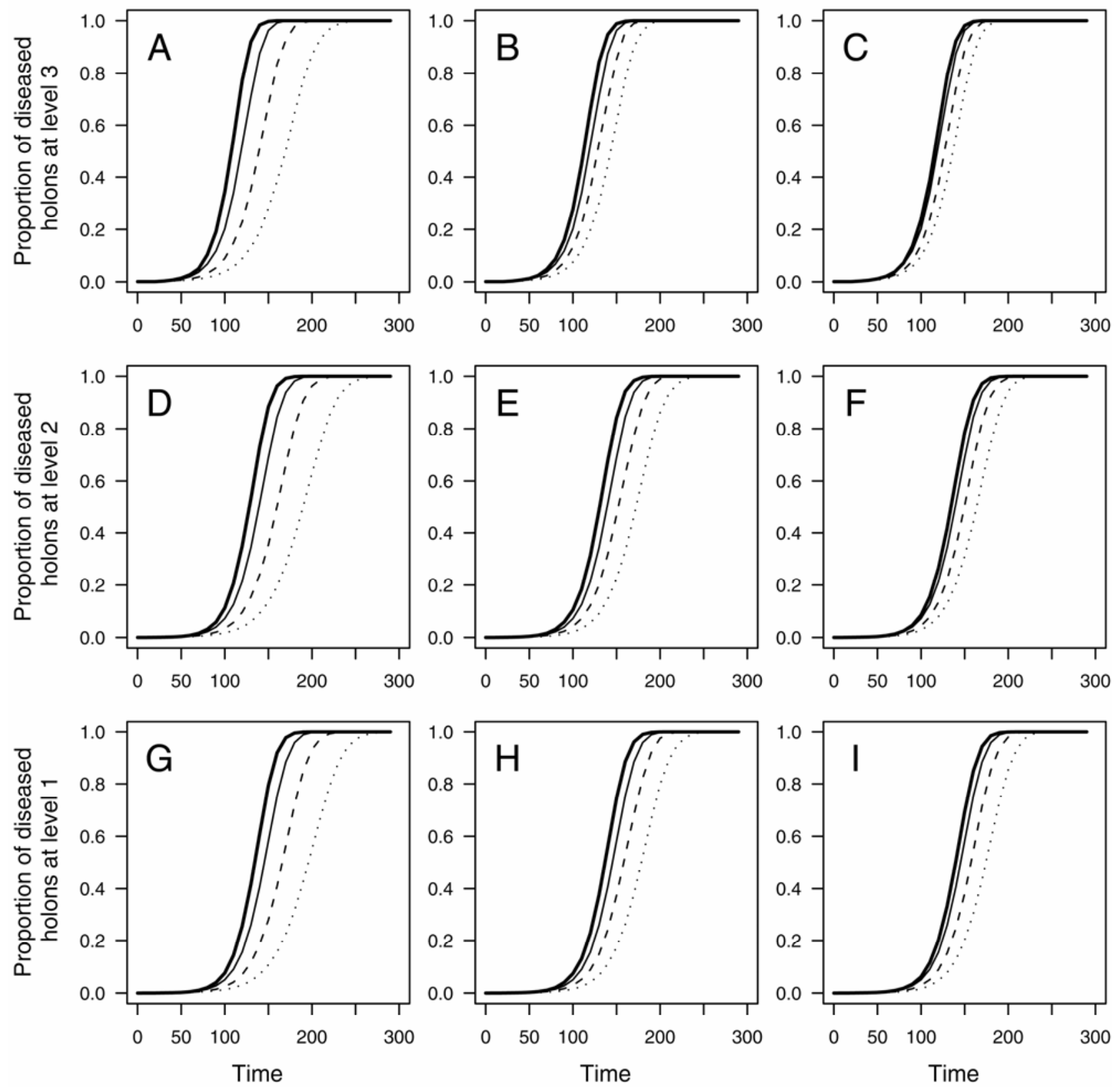

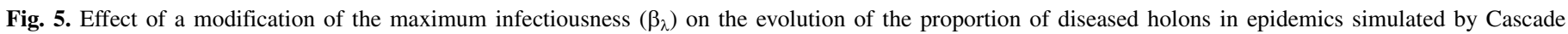

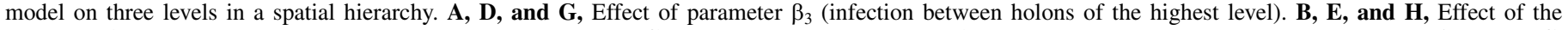

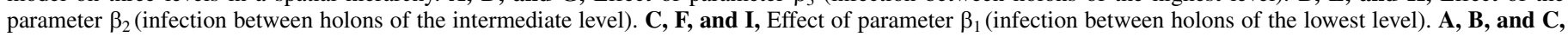

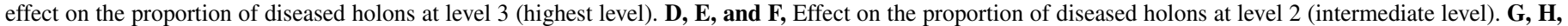

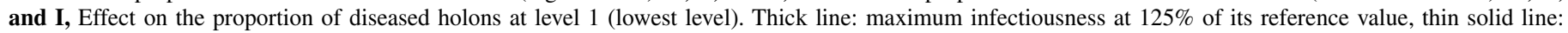
infectiousness at $100 \%$ of its reference value, dashed line: infectiousness at $75 \%$ of its reference value, dotted line: infectiousness at $50 \%$ of its reference value. 
population approach to more than two levels. As with any model, it is a simplification of reality and it is therefore wrong, but it captures features of epidemics that might be important and are not represented in other, non-hierarchical models such as the logistic model: for example, the fact that at any given level (e.g., site), individuals do not mix perfectly but tend to have more contacts with individuals from the same group (e.g., from the same leaf) than with individuals of different groups. As a result, the infection rate at the site level is higher than the infection rate at the leaf level, which leads to aggregation of the disease: it builds up within infected leaves before infecting new leaves, and within plants before infecting new plants and so on. Thus, the model describes epidemics in terms of three things: host population structure (definition of levels and number of holons at each level), maximum infectiousness at each level, and strength of coupling between "severity" and probability of infection. It allows the relationships between host spatial structure, differential infection rates between levels and predicted aggregation to be examined.

The sensitivity analyses performed on two sets of parameters (infectiousness of totally diseased holons and parameters of the link function between conditional incidence at one level and infectiousness at the level above) showed that these parameters had an effect both on the dynamics (Figs. 5 and 7) and on the spatial structure of the disease (Fig. 6; Tables 4 and 5), and that a modification of a parameter at any level had an effect on all levels in the spatial hierarchy.

The model outputs allowed aggregation of diseased holons to be characterized in two ways: first, through the relationship between incidences at two successive levels in the hierarchy (Fig. 3 ) and second, through the variance of the number of diseased

TABLE 2. Effect of the maximal infectiousness at each level $\left(\beta_{\lambda}\right)$ on the mean area under disease progress curve (standard deviation) at each level in epidemics simulated by Cascade model on three levels in a spatial hierarchy

\begin{tabular}{|c|c|c|c|c|c|c|c|c|c|}
\hline \multirow[b]{2}{*}{ Parameter value ${ }^{\mathrm{a}}$} & \multicolumn{3}{|c|}{ Effect of $\beta_{3}{ }^{b}$} & \multicolumn{3}{|c|}{ Effect of $\beta_{2}{ }^{b}$} & \multicolumn{3}{|c|}{ Effect of $\beta_{1}{ }^{b}$} \\
\hline & Level 3 & Level 2 & Level 1 & Level 3 & Level 2 & Level 1 & Level 3 & Level 2 & Level 1 \\
\hline $50 \%$ & $132.5(14)$ & $111.8(14)$ & $105.6(14)$ & $158.6(12)$ & $128.7(12)$ & $122.5(12)$ & $164.5(11)$ & $137.3(11)$ & $126.6(11)$ \\
\hline $75 \%$ & $161.9(11)$ & $141.2(11)$ & $135(11)$ & $173.1(10)$ & $149.2(10)$ & $143(10)$ & $175.2(10)$ & $152.2(10)$ & $144.5(10)$ \\
\hline $125 \%$ & $193.4(8)$ & $172.7(8)$ & $166.5(8)$ & $189(9)$ & $170.3(9)$ & $164.2(9)$ & $185.5(10)$ & $166.2(10)$ & $161(10)$ \\
\hline
\end{tabular}

${ }^{a}$ Reference values (100\%) were $2 \mathrm{E}-4,2 \mathrm{E}-2$, and $2 \mathrm{E}-2$ for $\beta_{3}, \beta_{2}$, and $\beta_{1}$, respectively.

${ }^{\mathrm{b}}$ Level $3=$ highest level, level $2=$ intermediate level, and level $1=$ lowest level.

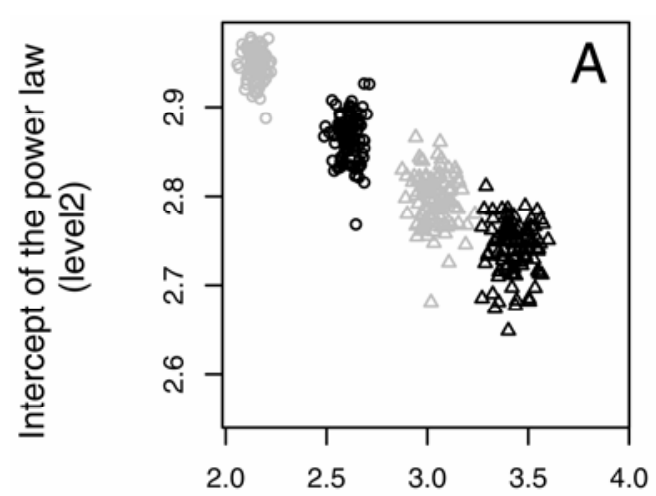

c (levels 3-2)

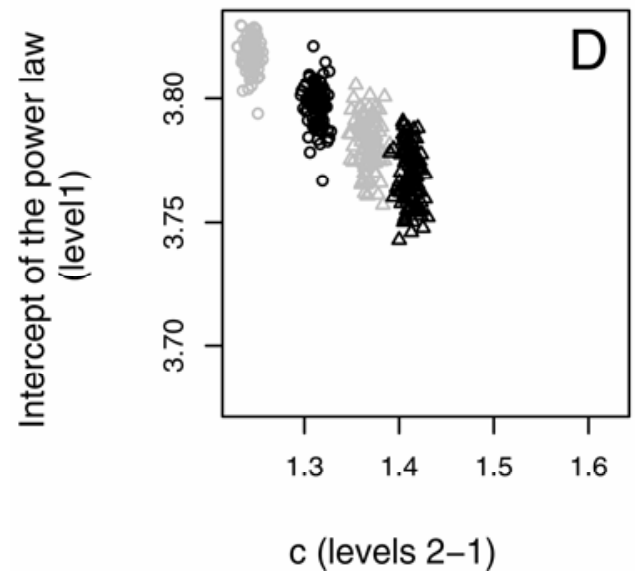

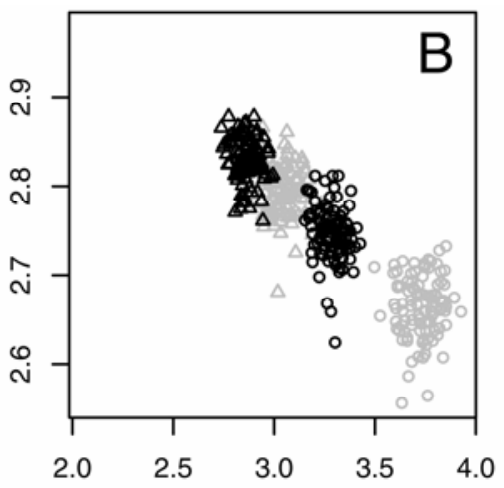

c (levels 3-2)

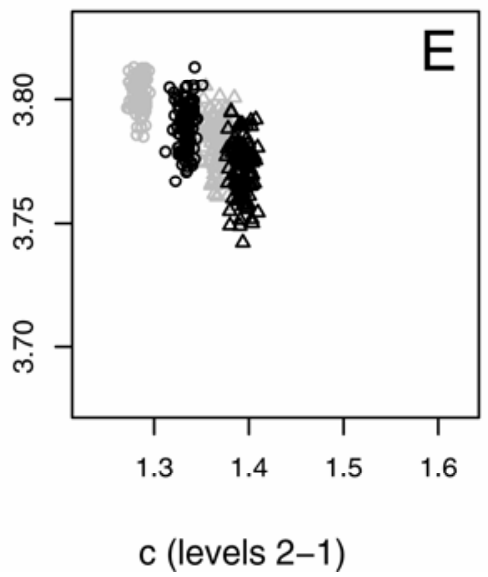

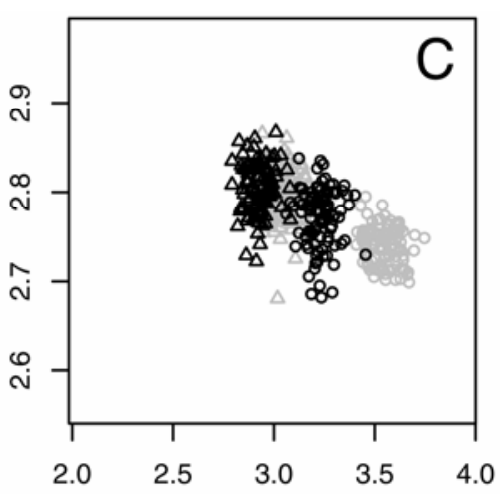

c (levels 3-2)

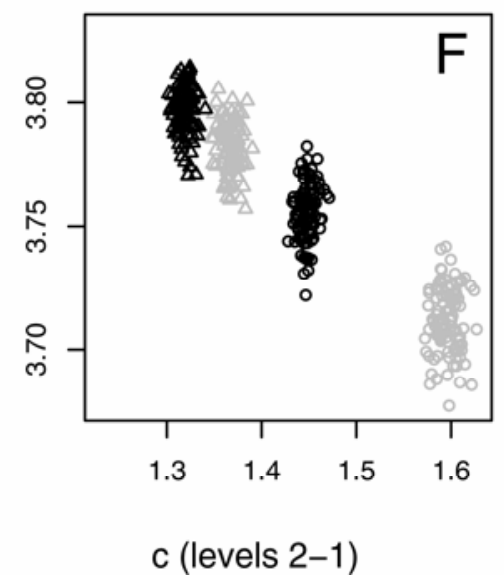

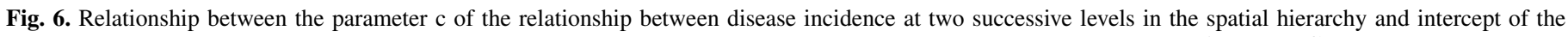

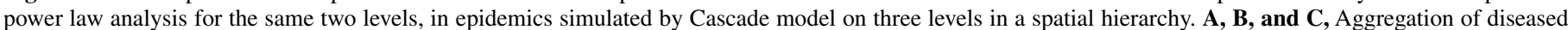

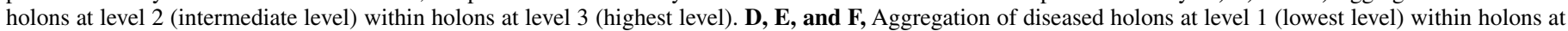

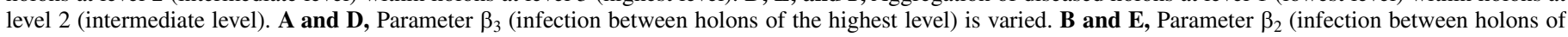

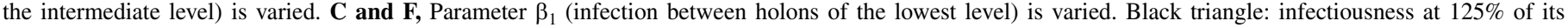

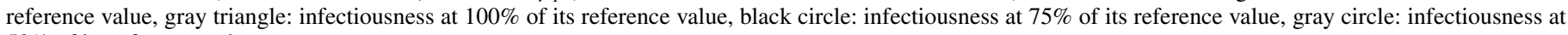
$50 \%$ of its reference value. 

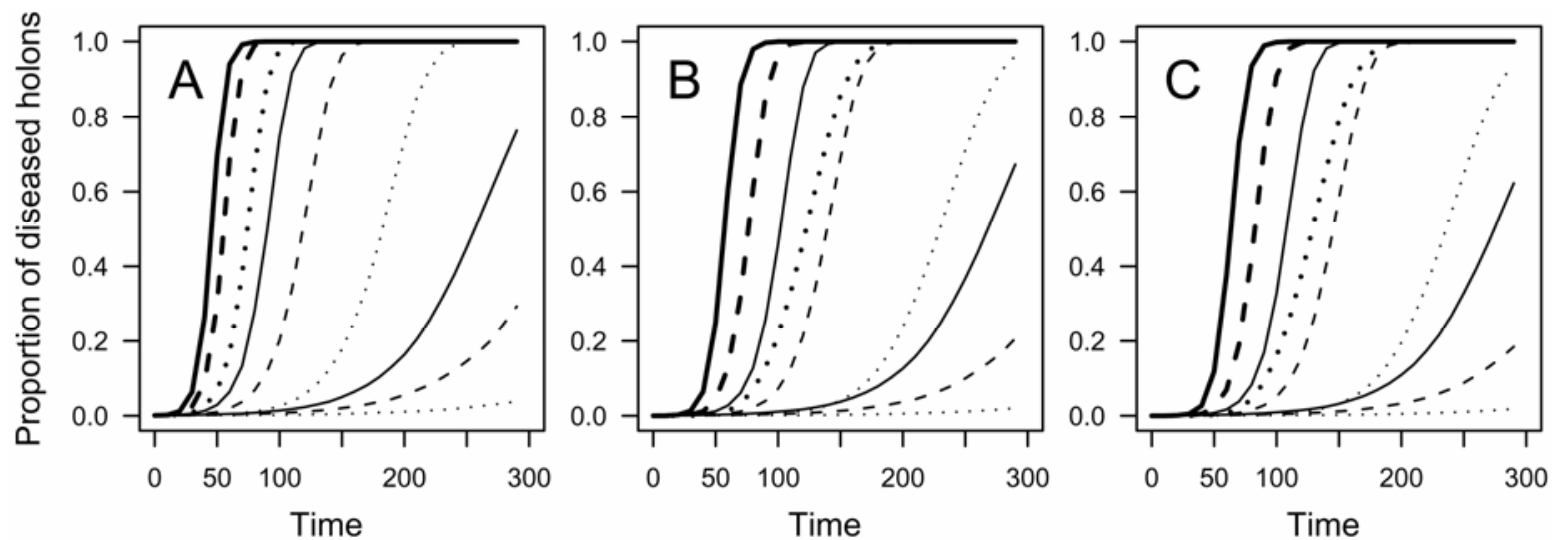

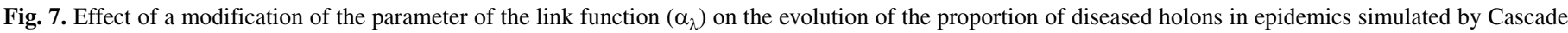

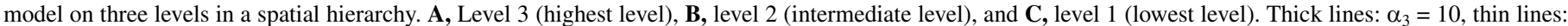
$\alpha_{3}=1$, gray lines: $\alpha_{3}=0.1$; solid line: $\alpha_{2}=10$, dashed line: $\alpha_{2}=1$ and dotted line: $\alpha_{2}=0.1$.

TABLE 3. Effect of the parameter of the link function on the area under disease progress curve (AUDPC) at each level in epidemics simulated by Cascade model on three levels in a spatial hierarchy

\begin{tabular}{lccc}
\hline Parameter values $\left(\alpha_{3} \alpha_{2}\right)^{\mathrm{a}}$ & $\begin{array}{c}\text { AUDPC } \\
\text { level 3 }\end{array}$ & $\begin{array}{c}\text { AUDPC } \\
\text { level 2 }\end{array}$ & $\begin{array}{c}\text { AUDPC } \\
\text { level } 1^{\mathrm{b}}\end{array}$ \\
\hline 0.10 .1 & $\begin{array}{c}3.4(1.5) \\
0.11\end{array}$ & $1.8(0.8)$ & $1.6(0.7)$ \\
0.110 & $20.0(7.6)$ & $13.9(5.6)$ & $12.4(5)$ \\
10.1 & $52.6(11.9)$ & $43.7(10.7)$ & $39.4(10)$ \\
11 & $121.2(12.6)$ & $73.2(12.2)$ & $67.3(12)$ \\
110 & $183.2(7.8)$ & $162.5(7.9)$ & $156.3(7.9)$ \\
100.1 & $210.2(7.9)$ & $198.4(7.9)$ & $192.2(7.9)$ \\
101 & $225.7(6.8)$ & $177.2(6.8)$ & $171.0(6.8)$ \\
1010 & $244.0(6)$ & $223.3(6)$ & $217.1(6)$ \\
\hline
\end{tabular}

${ }^{\mathrm{a}} \alpha_{3}=$ parameter of the link function between infectiousness at the highest level and conditional incidence at the intermediate level; $\alpha_{2}=$ parameter of the link function between infectiousness at the intermediate level and conditional incidence at the lowest level.

${ }^{\mathrm{b}}$ Mean value (standard deviation). Level $3=$ highest level, level $2=$ intermediate level, and level 1 = lowest level.

TABLE 4. Mean and standard deviation of the estimated c parameter of the relationship between disease incidences at two successive levels in the hierarchy $\left(\mathrm{I}_{\text {high }}=1-\left(1-\left(1-\mathrm{I}_{\text {low }}\right)\right)\right.$ as a function of the parameter of the link function in epidemics simulated by Cascade model on three levels in a spatial hierarchy

\begin{tabular}{lcccc}
\hline & & \multicolumn{4}{c}{$\alpha_{2}{ }^{\mathrm{c}}$} \\
\cline { 3 - 5 } Level $^{\mathrm{a}}$ & $\alpha_{3}{ }^{\mathrm{b}}$ & 0.1 & 1 & 10 \\
\hline $\mathrm{I}_{3}=\mathrm{f}\left(\mathrm{I}_{2}\right)$ & 0.1 & $1.89(0.11)$ & $1.49(0.02)$ & $1.31(0.01)$ \\
& 1 & $4.87(0.11)$ & $3.04(0.07)$ & $2.26(0.04)$ \\
& 10 & $15.25(0.47)$ & $7.86(0.28)$ & $4.26(0.18)$ \\
$\mathrm{I}_{2}=\mathrm{f}\left(\mathrm{I}_{1}\right)$ & 0.1 & $1.09(0.01)$ & $1.13(0.01)$ & $1.15(0.01)$ \\
& 1 & $1.20(0)$ & $1.37(0.01)$ & $1.51(0.01)$ \\
& 10 & $1.29(0)$ & $1.68(0.02)$ & $1.96(0.03)$ \\
\hline
\end{tabular}

a Relationships between incidences at the highest $\left(\mathrm{I}_{3}\right)$ and intermediate $\left(\mathrm{I}_{2}\right)$ levels, and between incidences at the intermediate $\left(\mathrm{I}_{2}\right)$ and lowest $\left(\mathrm{I}_{1}\right)$ levels.

b Parameter of the link function between infectiousness at the highest level and conditional incidence at the intermediate level.

c Parameter of the link function between infectiousness at the intermediate level and conditional incidence at the lowest level.

holons per group, which allowed a power law analysis (Fig. 4). Although somehow less numerically sensitive to differences in parameter values than the incidence-incidence relationship (there is more separation along the $\mathrm{x}$ axis than along the $\mathrm{y}$ axis in Figure $6)$, the power law analysis offers additional information. Indeed, four stages could be identified during the development of the epidemics based on the power law graph (Fig. 4). If one considers aggregation of diseased holons in groups, the initial increase in
TABLE 5. Mean and standard deviation of the intercept of the segment of the power law relationship corresponding to a constant level of aggregation (slope $=1$ ) as a function of the parameters of the link function in epidemics simulated by Cascade model on three levels in a spatial hierarchy

\begin{tabular}{lclll}
\hline & & \multicolumn{3}{c}{$\alpha_{2}{ }^{\mathrm{c}}$} \\
\cline { 3 - 5 } Level $^{\mathrm{a}}$ & $\alpha_{3}{ }^{\mathrm{b}}$ & \multicolumn{1}{c}{0.1} & \multicolumn{1}{c}{1} & 10 \\
\hline 2 & 0.1 & $3.02(0.108)$ & $3.12(0.007)$ & $3.15(0.005)$ \\
& 1 & $2.46(0.065)$ & $2.8(0.029)$ & $2.93(0.026)$ \\
& 10 & $1.46(0.06)$ & $2.35(0.05)$ & $2.69(0.037)$ \\
1 & 0.1 & $3.87(0.005)$ & $3.87(0.003)$ & $3.86(0.003)$ \\
& 1 & $3.83(0.006)$ & $3.78(0.01)$ & $3.75(0.015)$ \\
& 10 & $3.76(0.004)$ & $3.69(0.015)$ & $3.65(0.019)$ \\
\hline
\end{tabular}

${ }^{a}$ Level $2=$ variance of the number of infected holons at the intermediate level and level $1=$ variance of the number of infected holons at the lowest level.

${ }^{b}$ Parameter of the link function between infectiousness at the highest level and conditional incidence at the intermediate level.

c Parameter of the link function between infectiousness at the intermediate level and conditional incidence at the lowest level.

aggregation corresponds to the intensification of disease (holonto-holon infection) within previously infected groups while holons in healthy groups remain somewhat protected because group-togroup infection occurs more slowly. The second and third phases correspond to a period of constant aggregation (respectively, before and after the incidence at the holon level has reached 0.5); during these phases there is an equilibrium between the infection of new groups and the saturation of already-diseased groups, resulting in a stable "population pyramid" of diseased holons within nonsaturated diseased groups. The final decrease in disease aggregation corresponds to the progressive saturation of the lateinfected groups. This finding could not have been made without simulation: experimental data usually have too few points in time, particularly at the very beginning and very end of the epidemics, and also have too much uncertainty surrounding the estimated incidence values, to be able to detect the transition between the phases, which appeared sharply in the simulations when each repetition was observed individually. The observed behavior of the model is in agreement with empirical results and theoretical observations of the relationship between the mean and variance for aggregated disease incidence data (14). It is expected from theory, and has been observed in practice that the variance of the distribution of disease incidence should increase from low values at low incidence to reach a maximum around $P=0.5$ before declining at high disease incidence.

The parameters used in the sensitivity analysis were chosen because they both have an impact on the probability of infection. Obviously, increasing the maximum infectiousness increases the probability of infection. The parameter of the link function also 
has an effect on the probability of infection (equation 2): increasing the parameter $\alpha_{\lambda}$ makes the infectiousness less sensitive to the conditional incidence at the level below and so increases the probability of infection. However, these two parameters have very different biological meanings. Parameter $\beta$ represents the maximal efficiency of disease transmission, while parameter $\alpha$ represents the strength of coupling between disease intensity and disease transmission. The two extreme situations for the strength of coupling between two levels are a systemically transmitted pathogen and a pathogen with a high transmission threshold. A systemically transmitted pathogen (i.e., the holon becomes fully infective as soon as it is infected, whatever the number of diseased elements) will be represented in the model by a high value of $\alpha$; it could arise from a pathogen being systemic while symptoms appear progressively on different parts of a plant, or from a vector being particularly attracted to infected plants or plant parts. A high transmission threshold (i.e., a pathogen which can be transmitted only by severely attacked holons) will be represented in the model by a low value of $\alpha$; it could arise from a vector being attracted to healthy plants or plant parts; low values of $\alpha$ could also be used in order to introduce some sort of latency between the infection of a plant and its becoming fully infectious.

Whichever parameter was modified, the simulations of the model showed that increasing the infection rate at a given level increased aggregation of diseased holons at higher level(s) and decreased aggregation of diseased holons at lower level(s) (Tables 4 and 5; Fig. 6). Indeed, this model explains the existence of aggregation by the fact that some holons are "protected" from infection because they belong to uninfected groups and group-togroup infection occurs at a lower rate than holon-to-holon infection within a group (e.g., plant-to-plant infection within an infected field happens more often than infection between fields). In the case where the rate of infection at the highest level was too high compared to the rate of infection at the intermediate level (which happened when $\alpha_{3}=10$ and $\alpha_{2}=0.1$ ), aggregation was highly reduced (Tables 4 and 5). In this case, the model's assumptions may not hold. Indeed, the main assumption of the model is that once a group is infected, on one of its holons, infections of its holons only arise from secondary infections among these holons. This assumption holds only if interactions between holons from two different groups are negligible compared to interactions between holons of the same group (i.e., the rate of infection at one level is an order of magnitude lower than the rate of infection at the level below). For this reason, the definition of the levels is a crucial issue which must be given full consideration. Levels must be defined based on discontinuities in the spatial and temporal scales of the patterns and processes under study, which might not coincide with our own definitional classes, such as fields or plants $(4,13,39)$.

The model presented here considered only one type of disease spread (namely, perfect mixing). Nevertheless, the model could easily be modified to suit other cases: dispersion between nearest neighbors in one dimension, circular focal spread in two dimensions, or more complicated patterns. Indeed, the focal spread of diseases in two-dimensional space has been extensively studied, in particular the effect of the contact kernel (a function describing the probability that an infective propagule will reach a target location, depending on the distance between the source and the target) on the epidemic size (8) and on the shape and velocity of the front of the disease (10). The form of the mixing term for infection at the lower levels should have a small impact on the epidemic development if the maximal infectiousness is chosen so that the times to saturation are similar in all cases, and if the link function is linear $(\alpha=1)$. But if the shape of the saturation curve were different, and there were nonlinearities (e.g., thresholds) in the relationship between intensity of the disease at one level and probability of infection at the level above, the mixing term (representing the spatial characteristics of the infection process) would have a stronger effect on disease dynamics.

Another possible extension of the model would be to allow for the recovery or removal of diseased holons. Indeed the model presented here is an SI model; it is a useful tool to study the transient behavior of the system, but eventually, all holons at all scales will become infected. SIR or SIS models (with possible recovery-removal or return to a susceptible state) might exhibit different long-term behaviors (2,7). But the conception of such models would raise the question of the meaning of recovery or removal at each level: it could either happen at the holon level, and groups would then be considered removed when all of their holons are removed, or it could happen at the group level, and then all holons in a group would be immediately removed when the group is removed.

Even in its current form, the model proposed here might be applied to numerous pathosystems, as long as it is possible to define nonoverlapping hierarchical levels characterized by strong discontinuities in the rate of infection along the spatiotemporal scale. This situation is common for soilborne pathogens, e.g., in the case of take-all disease of wheat: lesion extension along roots (time scale = days) $(11)$, between-roots dispersal within a row (time scale $=$ weeks), between-rows dispersal (time scale = months) (37), between-field dispersal by contaminated material or ascospores (time scale $=$ years) $(1)$. This is also seen for aerial pathogens (both plant and animal pathogens) when spatial discontinuities are present, e.g., islands within an island chain, populations within an island (28), or when different infection processes occur at different spatial and temporal scales, e.g., infection between sheep within herds (time scale $=$ months) and infections between herds during the transhumance or through trading of diseased animals (time scale $=$ years) $(6,24)$. The range of hypotheses that this model could explore is also very wide: it could be used in order to test the effect of vector efficiency or the fact that a disease is systemic (through the link function); or the effect of host architecture, e.g., difference in spread between an oat panicle, a wheat ear and a corn cob (through dimensionality of infection); or the effect of host growth and spatial structure (through the type and rate of growth and maximum number of holons per group); or the effect of quantity and distribution of the primary inoculum (through number of initially infected holons at each level). For example, the effect of the host's spatial structure, and of the primary inoculum's quantity and distribution on disease spread has been studied using this model (12). The model presented here is thus a conceptual framework that can be used in order to address a number of epidemiological processes taking place within a spatial hierarchy.

\section{ACKNOWLEDGMENTS}

This research was partly supported by Institut National de la Recherche Agronomique, Agrocampus Rennes, and Région Bretagne. We thank S. Savary for the fruitful discussions on how to include several spatial scales in epidemiological models and L. Willocquet for her advice concerning the sensitivity analysis as well as the structure of the manuscript, and more generally her role as an advisor. We also acknowledge the helpful suggestions of the anonymous reviewers and the editor.

\section{LITERATURE CITED}

1. Asher, M. J. C., and Shipton, P. J. 1981. Page 538 in: Biology and Control of Take-All. Academic Press, London.

2. Bolker, B. M. 1999. Analytic models for the patchy spread of disease. B. Math. Biol. 61:849-874.

3. Bolker, B. M., and Pacala, S. W. 1997. Using moment equations to understand stochastically driven spatial pattern formation in ecological systems. Theor. Popul. Biol. 52:179-197.

4. Bragg, D. C., Roberts, D. W., and Crow, T. R. 2004. A hierarchical approach for simulating northern forest dynamics. Ecol. Model. 173: 31-94. 
5. Campbell, C. L., and Madden, L. V. 1990. Temporal analysis of epidemics II: Advanced topics. Pages 203-251 in: Introduction to Plant Disease Epidemiology. C. L. Campbell and L. V. Madden, eds. Wiley-Interscience, New York.

6. Durand, B., Dubois, M. A., Sabatier, P., Calavas, D., Ducrot, C., and van den Wielle, A. 2004. Multiscale modelling of scrapie epidemiology: II. Geographical level: Hierarchical transfer of the herd model to the regional disease spread. Ecol. Model. 179:515-531.

7. Filipe, J. A. N., and Maule, M. M. 2003. Analytical methods for predicting the behaviour of population models with general spatial interactions. Math. Biosci. 183:15-35.

8. Filipe, J. A. N, and Maule, M. M. 2004. Effects of dispersal mechanisms on spatio-temporal development of epidemics. J. Theor. Biol. 226:125141.

9. Filipe, J. A. N., Maule, M. M., and Gilligan, C. A. 2004. On "analytical models for the patchy spread of plant disease". Bull. Math. Biol. 66:10271037.

10. Frantzen, J., and van den Bosch, F. 2000. Spread of organisms: Can traveling and dispersive waves be distinguished? Basic Appl. Ecol. 1:8391.

11. Gilligan, C. A. 1980. Colonization of lateral, seminal and adventitious roots of wheat by the take-all fungus, Gaeumannomyces graminis var. tritici. J. Agric. Sci. 94:325-329.

12. Gosme, M., and Lucas, P. 2009. Disease spread across multiple scales in a spatial hierarchy: Effect of host spatial structure, and of inoculum quantity and repartition. Phytopathology. 99:833-839.

13. Hay, G. J., Dube, P., Bouchard, A., and Marceau, D. J. 2002. A scalespace primer for exploring and quantifying complex landscapes. Ecol. Model. 153:27-49.

14. Hughes, G., and Madden, L. V. 1993. Using the beta-binomial distribution to describe aggregated patterns of disease incidence. Phytopathology 83:759-763.

15. Hughes, G., and Gottwald, T. R. 1999. Survey methods for assessment of Citrus tristeza virus incidence when Toxoptera citricida is the predominant vector. Phytopathology 89:487-494.

16. Hughes, G., McRoberts, N., Madden, L. V., and Gottwald, T. R. 1997. Relationships between disease incidence at two levels in a spatial hierarchy. Phytopathology 87:542-550.

17. Kampmeijer, P., and Zadoks, J. C. 1977. EPIMUL: A simulator of foci and epidemics in mixtures of resistant and susceptible plants, mosaics and multilines. Page 50 in: Simulation Monographs. Centre for Agricultural Publishing and Documentation, Wageningen, Netherlands.

18. Koestler, A. 1967. The Ghost in the Machine. Hutchinson \& Co., London.

19. Madden, L. V., and Hughes, G. 1999. An effective sample size for predicting plant disease incidence in a spatial hierarchy. Phytopathology. 89:770-781.

20. Madden, L. V., Hughes, G., and Ellis, M. A. 1995. Spatial heterogeneity of grape downy mildew. Phytopathology 85:269-275.

21. McRoberts, N., Hughes, G., and Madden, L. V. 1996. Incorporatinspatial variability into simple disease progress models for crop pathogens. Pages 75-82 in: Aspects of Applied Biology. Modelling in Applied Biology: Spatial Aspects, Assoc. Appl. Biol., Wellesbourne, UK.

22. McRoberts, N., Hughes, G., and Madden, L. V. 2003. The theoretical and practical application of relationships between different disease intensity measurements in plants. Ann. Appl. Biol. 142:191-211.

23. Müller, J.-P., Ratzé, C., Gillet, F., and Stoffel, K. 2005. Modeling and simulating hierarchies using an agent-based approach. In: MODSIM 2005 International Congress on Modelling and Simulation. Modelling and Simulation Society of Australia and New Zealand, Melbourne.

24. Sabatier, P., Durand, B., Dubois, M. A., Ducrot, C., Calavas, D., and van den Wielle, A. 2004. Multiscale modeling of scrapie epidemiology: I Herd level: A discrete model of disease transmission in a sheep flock. Ecol. Model. 180:233-252.

25. Savary, S., Castilla, N. P., and Willocquet, L. 2001. Analysis of spatiotemporal structure of rice sheath blight epidemics in a farmer's field. Plant Pathol. 50:53-68.

26. Savary, S., Willocquet, L., and Teng, P. S. 1997. Modeling sheath blight epidemics on rice tillers. Agric. Syst. 55:359-384.

27. Scherm, H., Ngugi, H. K., and Ojiambo, P. S. 2006. Trends in theoretical plant epidemiology. Eur. J. Plant. Pathol. 115:61-73.

28. Smith, D. L., Ericson, L., and Burdon, J. J. 2003. Epidemiological patterns at multiple spatial scales: An 11-year study of a Triphragmium ulmariae-Filipendula ulmaria metapopulation. J. Ecol. 91:890-903.

29. Taylor, L. R. 1961. Aggregation, variance and the mean. Nature 189:732735 .

30. Turechek, W. W., and Madden, L. V. 1999. Spatial pattern analysis of strawberry leaf blight in perennial production systems. Phytopathology. 89:421-433.

31. Turechek, W. W., and Madden, L. V. 2001. Effect of scale on plant disease incidence and heterogeneity in a spatial hierarchy. Ecol. Model. 144:7795.

32. Turechek, W. W., and Madden, L. V. 2003. A generalized linear modeling approach for characterizing disease incidence in a spatial hierarchy. Phytopathology 93:458-466.

33. Van der Plank, J. E. 1963. Page 349 in: Plant Diseases: Epidemics and Control. Academic Press, New York.

34. Waggoner, P. E., and Rich, S. 1981. Lesion distribution, multiple infection and the logistic increase of plant disease. Proc. Natl. Acad. Sci. USA 78:3292-3295

35. Watts, D., Muhamad, R., Medina, D. C., and Dodds, P. S. 2005. Multiscale, resurgent epidemics in a hierarchical metapopulation model. Proc. Natl. Acad. Sci. USA 102:11157-11162.

36. Willocquet, L., Fernandez, L., and Savary, S. 2000. Effect of various crop establishment methods practiced by Asian farmers on epidemics of rice sheath blight caused by Rhizoctonia solani. Plant Pathol. 49:346-354

37. Willocquet, L., and Lebreton, L. 2005. Focal spread of wheat take-all disease. (Abstr.) Phytopathology 95(suppl.) S111.

38. Willocquet, L., and Savary, S. 2004. An epidemiological simulation model with three scales of spatial hierarchy. Phytopathology 94:883-891.

39. Wu, J. G., and David, J. L. 2002. A spatially explicit hierarchical approach to modeling complex ecological systems: Theory and applications. Ecol. Model. 153:7-26.

40. Xu, X.-M., and Ridout, M. S. 1996. Analysis of disease incidence data using a stochastic spatio-temporal simulation model. Asp. Appl. Biol. 46:155-158

41. Xu, X.-M., Parry, D. W., Edwards, S. G., Cooke, B. M., Doohan, F. M., Van Maanen, A., Brennan, J. M., Monaghan, S., Moretti, A., Tocco, G., Mule, G., Hornok, L., Giczey, G., Tatnell, J., Nicholson, P., and Ritieni, A. 2004. Relationship between the incidences of ear and spikelet infection of Fusarium ear blight in wheat. Eur. J. Plant Pathol. 110:959-971.

42. Yang, X. B., and TeBeest, D. O. 1992. Dynamic pathogen distribution and logistic increase of plant disease. Phytopathology 82:380-383. 